\title{
Robust, source-independent biases in children's use of socially and individually acquired information: supplemental material
}

\author{
Mark Atkinson, Elizabeth Renner, Bill Thompson, Gemma Mackintosh, \\ Dongjie Xie, Yanjie Su, and Christine A. Caldwell
}

\section{Proportion of repeats for Experiments 1-3}

Proportion of repeats for all Stage A (Experiments 1-3) and Stage B (Experiments 1-2) data is illustrated in Figure S1. See Figure 2 (bottom row) for proportion of repeats for only the first five problems of Stage A.

\section{Statistical model outputs}

All linear mixed effects analyses were performed using R (R Core Team, 2013) and Ime4 (Bates et al., 2013) with logit regression. The (binary) dependent variable in each analysis was either task success or repeating behaviour, and the fixed effects output for each model is in the tables below.

Where specified as fixed effects, the following variables were sum coded: source (Social condition as -1 ; Individual condition as 1 ), information type (unrewarded information as -1 ; rewarded information as 1 ), population (UK as -1 ; China as 1 ), and reward structure (-1 if the reward positions were reversed in the information and test trials, as in Experiment 3; 1 if they were congruent, as in Experiments 1-2). Age, problem number, and stage were centred, with age scaled to measure thousands of days.

The random effects structure for each model aimed to include by-participant random slopes for all fixed effects, including interactions, following Barr et al. (2013) and the aim to keep random effects structures "maximal" where possible. Where this resulted in non-convergent or singular fit models, random slopes were removed, followed by random intercepts where necessary, until a convergent, non-singular model was obtained (again, following Barr et al., 2013).

Where model outputs indicated a significant interaction effect involving source and age, we investigated the effect of source on the younger (less than 4 years old) and older children 


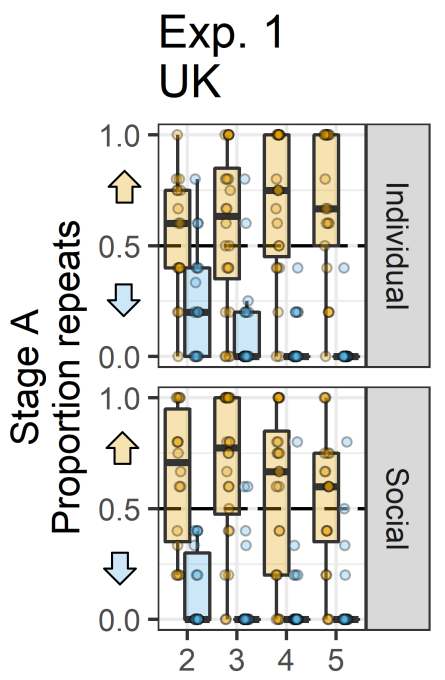

Exp. 2

China
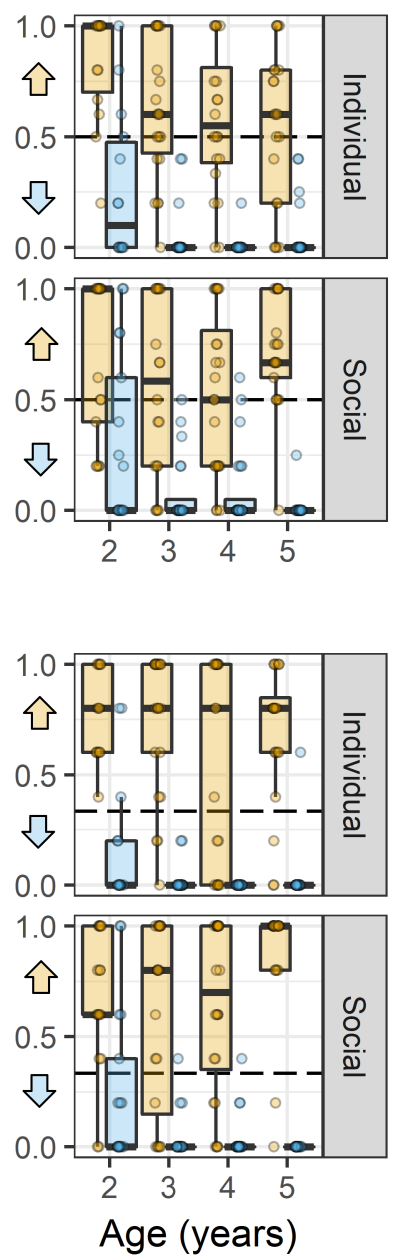

Exp. 3

UK (reversed)
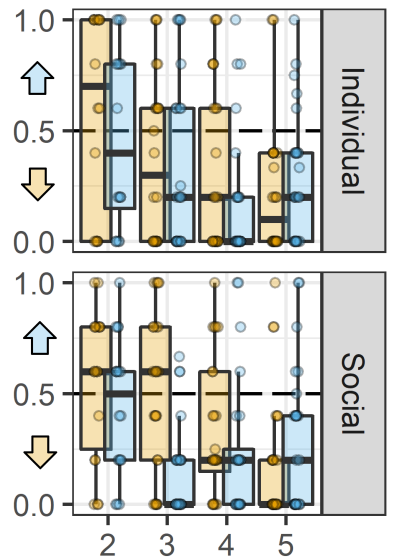

Info. type

审 Rewarded

Unrewarded

领 Direction of

论 task succes

Figure S1: Proportion of repeats by age (whole years) and information type for the Stage A (Experiments 1-3; top row) and Stage B (Experiments 1-2; bottom row) data. Proportion repeats arrows indicate whether repeats or non-repeats increase task success for a given experiment and information type, e.g. for Experiments 1-2, repeats following rewarded information trials increase task success, while non-repeats following unrewarded information trials increase task success (this pattern is reversed for Experiment 3). Dashed lines indicate chance performance. See Figure 1 (bottom row) for proportion repeats for only the first five problems of Stage A. 
( 4 years old and older) by rerunning the models (with the maximal model including age as a random effect rather than a fixed effect) on the two subsets of the data.

\subsection{Experiment 1}

\subsubsection{Task success for Stage A Problems 1-5}

Of the 172 children, 41 (24\%) located the reward in each of the first five problems of Stage A, and so completed no further Stage A problems. Seventeen [10\%] reached criterion after 6 problems, 11 [6\%] after 7 problems, 10 [6\%] after 8 problems, 12 [7\%] after 9 problems, and 9 [5\%] after 10 problems, while the remaining 72 [42\%] completed all 10 problems of Stage A without five consecutive successful TTs. For between-subject comparison purposes therefore, our analysis of task success considered only the first five problems of Stage A.

Model fixed effects were source, information type, age, and problem number, all interactions between source, information type, and age, and the interaction between source and problem number. Participant identity, experimenter identity, and participant gender were included as random intercepts. Model outputs are in Table S1.

Table S1: Experiment 1 task success for Stage A Problems 1-5.

\begin{tabular}{lrrrrr}
\hline & $b$ & $\mathrm{SE}(b)$ & \multicolumn{1}{c}{$\mathrm{z}$} & \multicolumn{1}{c}{$\mathrm{p}$} & \\
\hline Intercept & 1.438 & 0.154 & 9.335 & $<0.001$ & $* * *$ \\
source & 0.081 & 0.126 & 0.646 & 0.519 & \\
information type & -1.189 & 0.124 & -9.582 & $<0.001$ & $* * *$ \\
age & 1.061 & 0.309 & 3.434 & $<0.001$ & $* * *$ \\
problem number & 0.212 & 0.065 & 3.290 & 0.001 & $* *$ \\
source:information type & -0.121 & 0.116 & -1.046 & 0.295 & \\
source:age & 0.768 & 0.319 & 2.409 & 0.016 & $*$ \\
information type:age & -1.248 & 0.285 & -4.370 & $<0.001$ & $* * *$ \\
source:problem number & -0.044 & 0.064 & -0.684 & 0.494 & \\
source:information type:age & 0.211 & 0.282 & 0.746 & 0.456 & \\
\hline
\end{tabular}

As there was a significant interaction between source and age, we split our dataset into younger and older children based on median age, and repeated our analysis on these subsets. See Figure $\$ 2$ for mean task success by source for each age group.

For the younger children, model fixed effects were source, information type, problem number, and the interactions between source, and between information type and source and problem number. Participant identity and experimenter identity were included as random intercepts. Model outputs are in Table S2. 


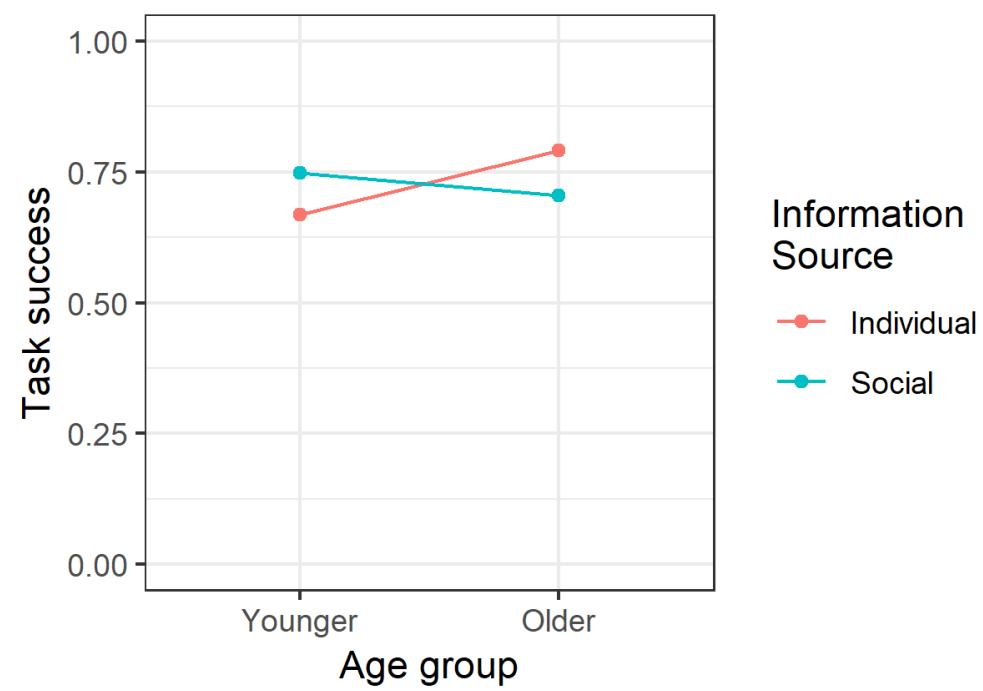

Figure S2: Experiment 1 mean task success by age group and source for Stage A Problems 1-5.

Table S2: Experiment 1 task success for Stage A Problems 1-5 for the younger children.

\begin{tabular}{lrrrrr}
\hline & $b$ & $\mathrm{SE}(b)$ & \multicolumn{1}{c}{$\mathrm{z}$} & \multicolumn{1}{c}{$\mathrm{p}$} & \\
\hline Intercept & 1.098 & 0.208 & 5.275 & $<0.001$ & $* * *$ \\
source & -0.188 & 0.138 & -1.368 & 0.171 & \\
information type & -0.853 & 0.131 & -6.516 & $<0.001$ & $* * *$ \\
problem number & 0.172 & 0.084 & 2.039 & 0.041 & $*$ \\
source:information type & -0.156 & 0.127 & -1.234 & 0.217 & \\
source:problem number & -0.051 & 0.084 & -0.603 & 0.547 & \\
\hline
\end{tabular}

For the older children, model fixed effects were source, information type, problem number, and the interactions between source and information type, and between source and problem number. Age was included as a random intercept. Model outputs are in Table S3.

Table S3: Experiment 1 task success for Stage A Problems 1-5 for the older children.

\begin{tabular}{lrrrrc}
\hline & $b$ & $\mathrm{SE}(b)$ & \multicolumn{1}{c}{$\mathrm{z}$} & \multicolumn{1}{c}{$\mathrm{p}$} & \\
\hline Intercept & 1.688 & 0.223 & 7.580 & $<0.001$ & $* * *$ \\
source & 0.360 & 0.193 & 1.872 & 0.061 &. \\
information type & -1.383 & 0.186 & -7.445 & $<0.001$ & $* * *$ \\
problem number & 0.230 & 0.098 & 2.352 & 0.019 & $*$ \\
source:information type & 0.008 & 0.165 & 0.048 & 0.962 & \\
source:problem number & -0.019 & 0.097 & -0.198 & 0.843 & \\
\hline
\end{tabular}




\subsubsection{Task success for Stage B}

For Stage B, we modified our definition of task success: 1 if the child's test trial selection gave a non-zero chance of locating the reward; 0 otherwise. This is still equivalent to a child's behaviour as if they had adopted a win-stay, lose-shift strategy (and under this definition task success scores in Stage A remain the same), but takes into account the effect of there being three stimuli. While a repeat selection following a rewarded information trial would locate the reward, we would expect non-repeat selections following an unrewarded information trial to locate the reward $50 \%$ of the time on average; such selections were still coded 1 for task success.

Model fixed effects were source, information type, age, and problem number, all interactions between source, information type, and age, and the interaction between source and problem number. Participant identity, experimenter identity, and participant gender were included as random intercepts, with by-participant random slopes for information type and problem number. Model outputs are in Table 54

Table S4: Experiment 1 task success for Stage B.

\begin{tabular}{lrrrrr}
\hline & $b$ & $\mathrm{SE}(b)$ & \multicolumn{1}{c}{$\mathrm{z}$} & \multicolumn{1}{c}{$\mathrm{p}$} & \\
\hline Intercept & 3.564 & 0.882 & 4.042 & $<0.001$ & $* * *$ \\
source & 0.095 & 0.189 & 0.501 & 0.616 & \\
information type & -1.934 & 0.360 & -5.376 & $<0.001$ & $* * *$ \\
age & 1.926 & 0.511 & 3.768 & $<0.001$ & $* * *$ \\
problem number & 0.015 & 0.037 & 0.405 & 0.686 & \\
source:information type & 0.211 & 0.175 & 1.205 & 0.228 & \\
source:age & 0.486 & 0.481 & 1.010 & 0.313 & \\
information type:age & -1.414 & 0.470 & -3.007 & 0.003 & $* *$ \\
source:problem number & -0.053 & 0.032 & -1.674 & 0.094 &. \\
source:information type:age & -0.073 & 0.440 & -0.165 & 0.869 & \\
\hline
\end{tabular}

The pattern of results for task success in the Stage B data is comparable to that of Stage A Problems $1-5$. Task success was above chance $(p<.001)$. There was no effect of source ( $p$ $=.616$ ). Task success was greater following unrewarded ITs compared to unrewarded ITs ( $p$ $<.001)$, and this was more pronounced in older children $(p=.003)$. Performance improved with age $(p<.001)$. Unlike in the Stage A Problems $1-5$ data, however, there is no effect of problem number $(p=.686)$, nor an interaction effect between source and age $(p=.313)$.

\subsubsection{Repeating behaviour for Stage A and Stage B}

Our fixed effects were source, information type, age, stage, and all interactions. Participant identity, experimenter identity, participant gender, and problem number were included as ran- 
dom intercepts, with by-participant random slopes for information type. Model outputs are in Table 55 ,

Table S5: Experiment 1 repeating behaviour for Stage A and Stage B.

\begin{tabular}{lrrrrr}
\hline & $b$ & $\mathrm{SE}(b)$ & \multicolumn{1}{c}{$\mathrm{z}$} & \multicolumn{1}{c}{$\mathrm{p}$} & \\
\hline Intercept & -1.454 & 0.248 & -5.864 & $<0.001$ & $* * *$ \\
source & 0.092 & 0.122 & 0.748 & 0.454 & \\
information type & 2.174 & 0.156 & 13.917 & $<0.001$ & $* * *$ \\
age & -1.218 & 0.315 & -3.864 & $<0.001$ & $* * *$ \\
stage & -0.048 & 0.145 & -0.333 & 0.739 & \\
source:information type & 0.064 & 0.119 & 0.540 & 0.589 & \\
source:age & 0.026 & 0.310 & 0.085 & 0.933 & \\
information type:age & 1.466 & 0.309 & 4.743 & $<0.001$ & $* * *$ \\
source:stage & 0.185 & 0.145 & 1.277 & 0.202 & \\
information type:stage & 0.439 & 0.145 & 3.028 & 0.002 & $* *$ \\
age:stage & 0.276 & 0.340 & 0.812 & 0.417 & \\
source:information type:age & 0.589 & 0.297 & 1.981 & 0.048 & $*$ \\
source:information type:stage & 0.011 & 0.145 & 0.077 & 0.938 & \\
source:age:stage & 0.124 & 0.341 & 0.364 & 0.716 & \\
information type:age:stage & 0.341 & 0.340 & 1.001 & 0.317 & \\
source:information type:age:stage & -0.400 & 0.341 & -1.175 & 0.240 & \\
\hline
\end{tabular}

As there was a significant interaction between source, information type, and age, we split our dataset into younger and older children based on median age, and repeated our analysis on these subsets. See Figure $\$ 3$ for mean proportion repeats by source and information type for each age group.

For the younger children, model fixed effects were source, information type, stage, and all interactions. Participant identity, participant gender, and age were included as random intercepts. Model outputs are in Table 56

Table S6: Experiment 1 repeating behaviour for Stage A and Stage B for the younger children.

\begin{tabular}{lrrrrr}
\hline & $b$ & $\mathrm{SE}(b)$ & \multicolumn{1}{c}{$\mathrm{z}$} & \multicolumn{1}{c}{$\mathrm{p}$} & \\
\hline Intercept & -0.870 & 0.161 & -5.400 & $<0.001$ & $* * *$ \\
source & 0.071 & 0.136 & 0.521 & 0.602 & \\
information type & 1.329 & 0.079 & 16.777 & $<0.001$ & $* * *$ \\
stage & -0.076 & 0.148 & -0.515 & 0.607 & \\
source:information type & -0.146 & 0.076 & -1.920 & 0.055 &. \\
source:stage & 0.150 & 0.148 & 1.012 & 0.311 & \\
information type:stage & 0.328 & 0.146 & 2.250 & 0.024 & $*$ \\
source:information type:stage & 0.251 & 0.146 & 1.720 & 0.085 &. \\
\hline
\end{tabular}




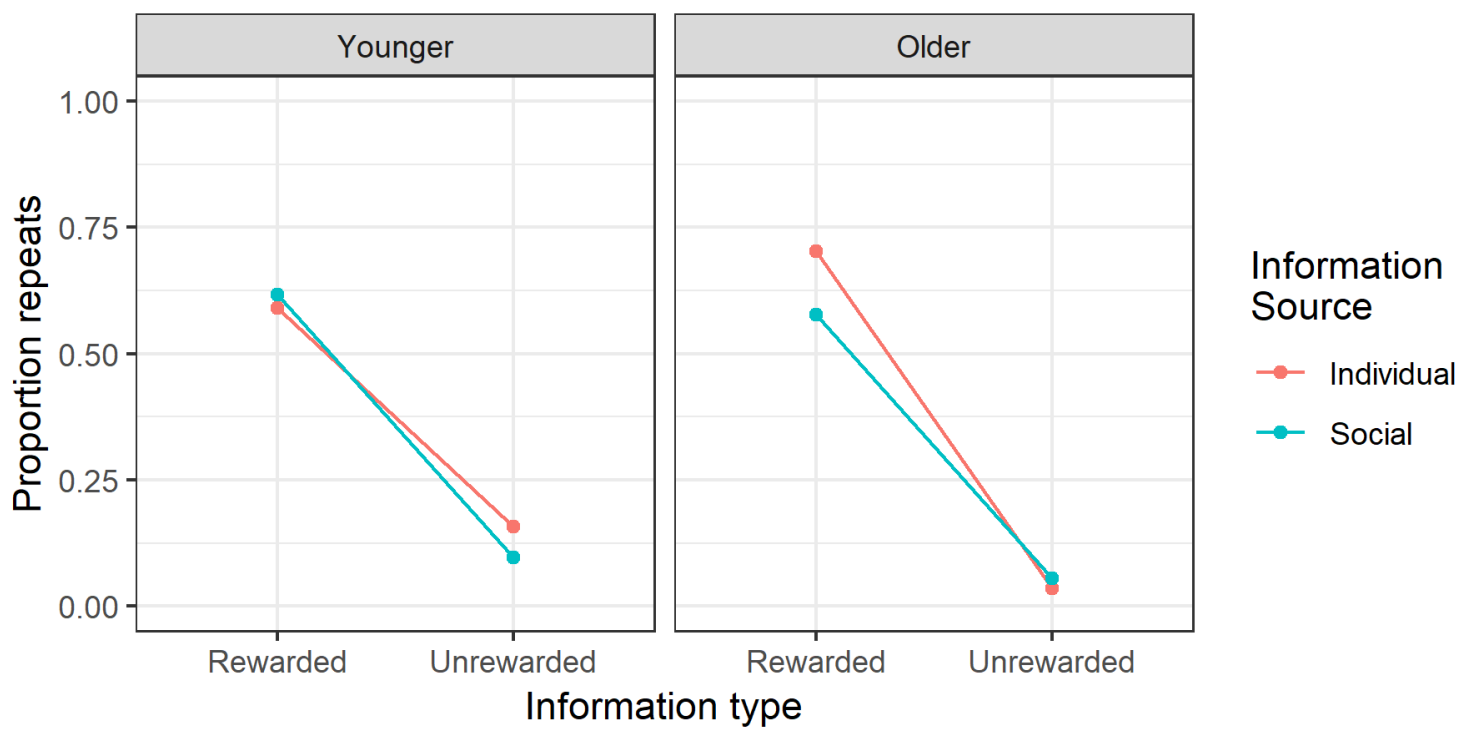

Figure S3: Experiment 1 mean proportion repeats for Stage A and Stage B by age group, source, and information type.

For the older children, model fixed effects were source, information type, stage, and all interactions. Participant identity, participant gender, problem number, experimenter identity, and age were included in random intercepts. Model outputs are in Table S7.

Table S7: Experiment 1 repeating behaviour for Stage A and Stage B for the older children.

\begin{tabular}{lrrrrr}
\hline & $b$ & $\mathrm{SE}(b)$ & \multicolumn{1}{c}{$\mathrm{z}$} & \multicolumn{1}{c}{$\mathrm{p}$} & \\
\hline Intercept & -1.364 & 0.340 & -4.016 & $<0.001$ & $* * *$ \\
source & 0.098 & 0.169 & 0.582 & 0.561 & \\
information type & 2.124 & 0.116 & 18.342 & $<0.001$ & $* * *$ \\
stage & -0.150 & 0.201 & -0.743 & 0.458 & \\
source:information type & 0.251 & 0.108 & 2.316 & 0.021 & $*$ \\
source:stage & 0.150 & 0.200 & 0.751 & 0.453 & \\
information type:stage & 0.717 & 0.200 & 3.582 & $<0.001$ & $* * *$ \\
source:information type:stage & -0.130 & 0.199 & -0.650 & 0.515 & \\
\hline
\end{tabular}

\subsection{Experiment 2}

Note that, unlike in the analyses above, there are no random intercepts for experimenter identity in the Experiment 2 analyses as all the data was collected by the same researcher.

\subsubsection{Task success for Stage A Problems 1-5}

Of the 159 children, $40(25 \%)$ located the reward in each of the first five problems of Stage A, and so completed no further Stage A problems. 22 [14\%] reached criterion after 6 problems, 
10 [6\%] after 7 problems, 13 [6\%] after 8 problems, 9 [6\%] after 9 problems, and 3 [2\%] after 10 problems, while the remaining 62 [39\%] completed all 10 problems of Stage A without five consecutive successful TTs. As in Experiment 1, our analysis of task success considered only the first five problems of Stage A.

Model fixed effects were source, information type, age, and problem number, all interactions between source, information type, and age, and the interaction between source and problem number. Participant identity was included as random intercepts. Model outputs are in Table 58 .

Table S8: Experiment 2 task success for Stage A Problems 1-5.

\begin{tabular}{lrrrrr}
\hline & $b$ & $\mathrm{SE}(b)$ & \multicolumn{1}{c}{$\mathrm{z}$} & $\mathrm{p}$ & \\
\hline Intercept & 1.631 & 0.162 & 10.082 & $<0.001$ & $* * *$ \\
source & 0.000 & 0.147 & -0.001 & 0.999 & \\
information type & -1.244 & 0.140 & -8.884 & $<0.001$ & $* * *$ \\
age & 0.953 & 0.379 & 2.515 & 0.012 & $*$ \\
problem number & 0.362 & 0.073 & 4.926 & $<0.001$ & $* * *$ \\
source:information type & -0.032 & 0.128 & -0.252 & 0.801 & \\
source:age & -0.341 & 0.380 & -0.898 & 0.369 & \\
information type:age & -1.745 & 0.333 & -5.242 & $<0.001$ & $* * *$ \\
source:problem number & 0.009 & 0.072 & 0.127 & 0.899 & \\
source:information type:age & -0.046 & 0.326 & -0.140 & 0.888 & \\
\hline
\end{tabular}

\subsubsection{Task success for Stage B}

Model fixed effects were source, information type, age, and problem number, all interactions between source, information type, and age, and the interaction between source and problem number. Participant identity was included as a random intercept. Model outputs are in Table 59 .

As for the Stage A Problems 1-5 data, task success was significantly above chance $(p<.001)$. There was no overall effect of source $(p=.824)$. Task success was greater following unrewarded ITs compared to rewarded ITs $(p<.001)$, and this was more pronounced in older children ( $p$ $<.001)$. Performance improved with problem number $(p=.010)$ and with age $(p<.001)$.

Unlike in the Stage A Problems 1-5 data, there was an interaction between source and age $(p=.039)$. We split our dataset into younger and older children based on median age, and repeated our analysis with these subsets. See Figure $\$ 4$ for mean task success by source for each age group. 
Table S9: Experiment 2 task success for Stage B.

\begin{tabular}{lrrrrc}
\hline & $b$ & $\mathrm{SE}(b)$ & \multicolumn{1}{c}{$\mathrm{z}$} & $\mathrm{p}$ & \\
\hline Intercept & 3.095 & 0.251 & 12.344 & $<0.001$ & $* * *$ \\
source & -0.048 & 0.218 & -0.222 & 0.824 & \\
information type & -1.811 & 0.167 & -10.878 & $<0.001$ & $* * *$ \\
age & 2.872 & 0.569 & 5.045 & $<0.001$ & $* * *$ \\
problem number & 0.077 & 0.030 & 2.591 & 0.010 & $*$ \\
source:information type & 0.009 & 0.157 & 0.055 & 0.956 & \\
source:age & -1.145 & 0.554 & -2.065 & 0.039 & $*$ \\
information type:age & -2.471 & 0.389 & -6.344 & $<0.001$ & $* * *$ \\
source:problem number & 0.022 & 0.030 & 0.745 & 0.456 & \\
source:information type:age & -0.020 & 0.376 & -0.054 & 0.957 & \\
\hline
\end{tabular}

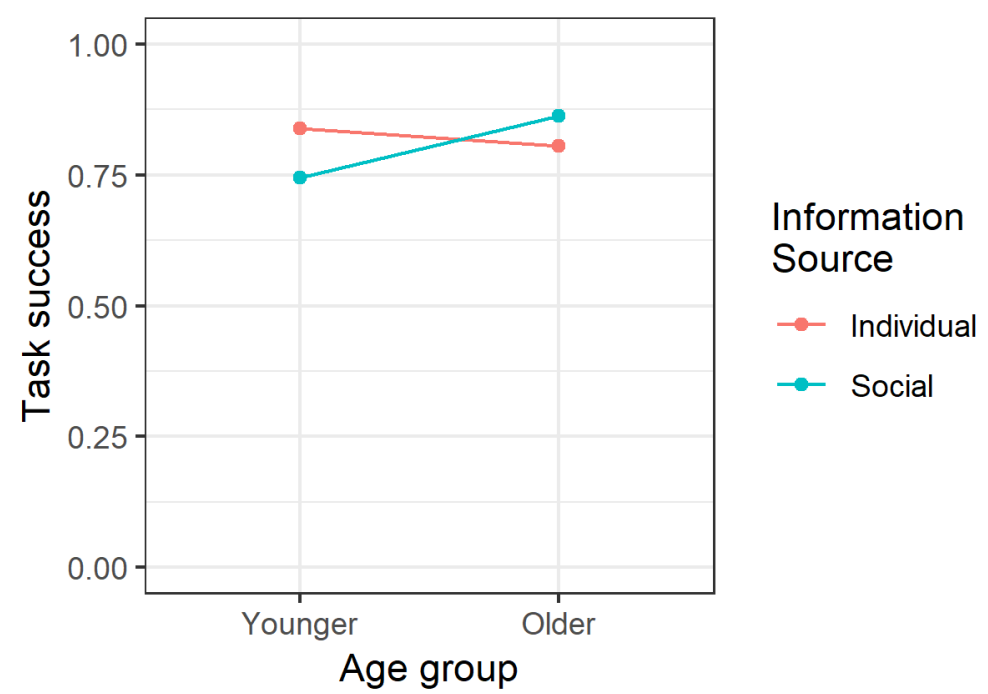

Figure S4: Experiment 2 mean task success by age group and source for Stage B.

For the younger children, model fixed effects were source, information type, problem number, and the interactions between source and information type, and between source and problem number. Participant identity and age were included as random intercepts. Model outputs are in Table $\mathrm{S10}$.

For the older children, model fixed effects were source, information type, problem number, and the interactions between source and information type, and between source and problem number. Participant identity and age were included as random intercepts. Model outputs are in Table S11.

The trends were in opposite directions, although the effect of source was only significant in the younger $(p=.045)$, and not the older $(p=.252)$, children. 
Table S10: Experiment 2 task success for Stage B for the younger children.

\begin{tabular}{lrrrrc}
\hline & $b$ & $\mathrm{SE}(b)$ & $\mathrm{z}$ & $\mathrm{p}$ & \\
\hline Intercept & 1.773 & 0.179 & 9.880 & $<0.001$ & $* * *$ \\
source & 0.338 & 0.169 & 2.005 & 0.045 & $*$ \\
information type & -0.730 & 0.113 & -6.459 & $<0.001$ & $* * *$ \\
problem number & 0.078 & 0.036 & 2.137 & 0.033 & $*$ \\
source:information type & -0.010 & 0.111 & -0.087 & 0.931 & \\
source:problem number & 0.042 & 0.036 & 1.160 & 0.246 & \\
\hline
\end{tabular}

Table S11: Experiment 2 task success for Stage B for the older children.

\begin{tabular}{lrrrrr}
\hline & $b$ & $\mathrm{SE}(b)$ & \multicolumn{1}{c}{$\mathrm{z}$} & \multicolumn{1}{c}{$\mathrm{p}$} & \\
\hline Intercept & 4.547 & 0.553 & 8.228 & $<0.001$ & $* * *$ \\
source & -0.505 & 0.440 & -1.146 & 0.252 & \\
information type & -2.800 & 0.316 & -8.861 & $<0.001$ & $* * *$ \\
problem number & 0.088 & 0.054 & 1.650 & 0.099 &. \\
source:information type & -0.063 & 0.291 & -0.216 & 0.829 & \\
source:problem number & -0.021 & 0.053 & -0.393 & 0.695 & \\
\hline
\end{tabular}

\subsubsection{Repeating behaviour for Stage A and Stage B}

Our fixed effects were source, information type, age, stage, and all interactions. Participant identity, participant gender, and problem number were included as a random intercept, with by-participant random slopes for information type and stage. Model outputs are in Table S12.

\subsection{Experiments $1-2$ combined data}

\subsubsection{Task success for Stage A Problems 1-5}

Our fixed effects were source, information type, age, problem number, population, all interactions between source, information type, age, and population, and all interactions between source, problem number, and population. Participant identity was included as a random intercept. Model outputs are in Table \$13.

As there was a significant interaction between source, age, and population, we split our dataset into younger and older children based on median age and repeated out analysis on these subsets. See Figure $\mathrm{S} 5$ for mean task success by source and population for each age group.

For the younger children, model fixed effects were source, information type, population, and problem number, all interaction between source, information type, and population, and all interactions between source, problem number, and population. Participant identity was included as random intercepts. Model outputs are shown in Table S14. 
Table S12: Experiment 2 repeating behaviour for Stage A and Stage B.

\begin{tabular}{lrrrrr}
\hline & $b$ & $\mathrm{SE}(b)$ & \multicolumn{1}{c}{$\mathrm{z}$} & \multicolumn{1}{c}{$\mathrm{p}$} & \\
\hline Intercept & -2.361 & 0.451 & -5.237 & $<0.001$ & $* * *$ \\
source & -0.084 & 0.219 & -0.384 & 0.701 & \\
information type & 3.429 & 0.378 & 9.071 & $<0.001$ & $* * *$ \\
age & -1.887 & 0.587 & -3.216 & 0.001 & $* *$ \\
stage & -0.446 & 0.244 & -1.831 & 0.067 &. \\
source:information type & 0.100 & 0.193 & 0.517 & 0.605 & \\
source:age & -0.029 & 0.586 & -0.049 & 0.961 & \\
information type:age & 1.658 & 0.515 & 3.217 & 0.001 & $* *$ \\
source:stage & -0.057 & 0.208 & -0.276 & 0.782 & \\
information type:stage & 1.227 & 0.231 & 5.308 & $<0.001$ & $* * *$ \\
age:stage & 0.235 & 0.510 & 0.460 & 0.645 & \\
source:information type:age & -0.981 & 0.515 & -1.904 & 0.057 &. \\
source:information type:stage & -0.093 & 0.198 & -0.468 & 0.640 & \\
source:age:stage & -0.134 & 0.508 & -0.263 & 0.793 & \\
information type:age:stage & 0.814 & 0.486 & 1.674 & 0.094 &. \\
source:information type:age:stage & -0.474 & 0.470 & -1.008 & 0.313 & \\
\hline
\end{tabular}

Table S13: Experiment 1-2 combined data task success for Stage A Problems 1-5.

\begin{tabular}{lrrrrrl}
\hline & $b$ & $\mathrm{SE}(b)$ & \multicolumn{1}{c}{$\mathrm{z}$} & $\mathrm{p}$ & \\
\hline Intercept & 1.525 & 0.104 & 14.664 & $<0.001$ & $* * *$ \\
source & 0.050 & 0.096 & 0.517 & 0.605 & \\
information type & -1.211 & 0.093 & -13.062 & $<0.001$ & $* * *$ \\
age & 1.009 & 0.241 & 4.181 & $<0.001$ & $* * *$ \\
population & 0.045 & 0.096 & 0.470 & 0.638 & \\
problem number & 0.569 & 0.097 & 5.861 & $<0.001$ & $* * *$ \\
source:information type & -0.077 & 0.086 & -0.891 & 0.373 & \\
source:age & 0.243 & 0.241 & 1.009 & 0.313 & \\
information type:age & -1.486 & 0.218 & -6.823 & $<0.001$ & $* * *$ \\
source:population & -0.045 & 0.096 & -0.468 & 0.640 & \\
information type:population & 0.026 & 0.086 & 0.299 & 0.765 & \\
age:population & -0.066 & 0.241 & -0.274 & 0.784 & \\
source:problem number & -0.031 & 0.095 & -0.329 & 0.742 & \\
population:problem number & 0.128 & 0.096 & 1.343 & 0.179 & \\
source:information type:age & 0.080 & 0.215 & 0.373 & 0.710 & \\
source:information type:population & 0.044 & 0.086 & 0.507 & 0.612 & \\
source:age:population & -0.558 & 0.242 & -2.305 & 0.021 & $*$ \\
information type:age:population & -0.208 & 0.215 & -0.967 & 0.334 & \\
source:population:problem number & 0.053 & 0.095 & 0.555 & 0.579 & \\
source:information type:age:population & -0.133 & 0.215 & -0.620 & 0.535 & \\
\hline
\end{tabular}

For the older children, model fixed effects were source, information type, population, and problem number, all interaction between source, information type, and population, and all 


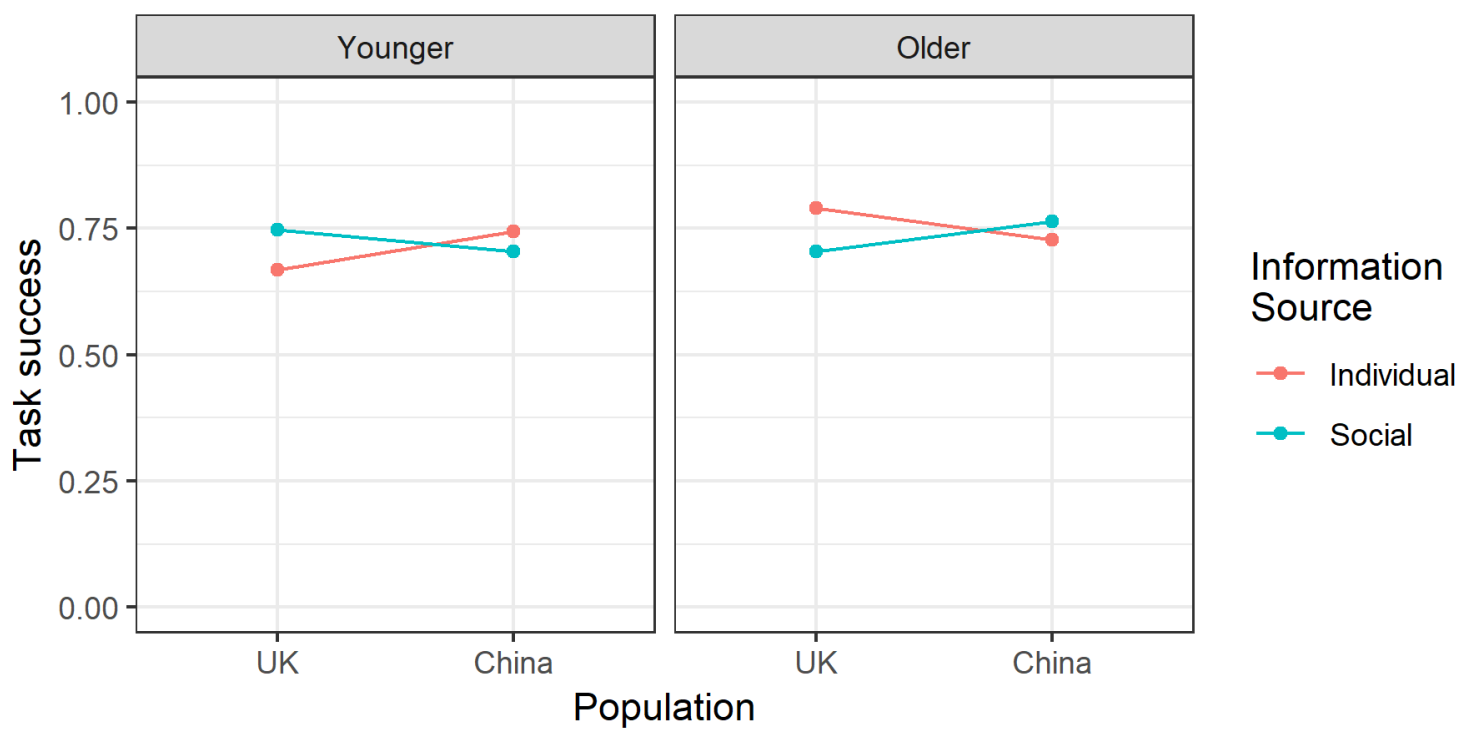

Figure S5: Experiments 1-2 mean task success for Stage A Problems 1-5 by age group, source, and population.

Table S14: Experiment 1-2 combined data task success for Stage A Problems 1-5 for the younger children.

\begin{tabular}{lrrrrr} 
& $b$ & $\mathrm{SE}(b)$ & \multicolumn{1}{c}{$\mathrm{z}$} & \multicolumn{1}{c}{$\mathrm{p}$} & \\
\hline Intercept & 1.107 & 0.111 & 10.010 & $<0.001$ & $* * *$ \\
source & -0.061 & 0.104 & -0.586 & 0.558 & \\
information type & -0.684 & 0.095 & -7.224 & $<0.001$ & $* * *$ \\
population & -0.003 & 0.104 & -0.034 & 0.973 & \\
problem number & 0.179 & 0.063 & 2.856 & 0.004 & $* *$ \\
source:information type & -0.110 & 0.092 & -1.200 & 0.230 & \\
source:population & 0.187 & 0.104 & 1.797 & 0.072 &. \\
information type:population & 0.199 & 0.092 & 2.168 & 0.030 & $*$ \\
source:problem number & -0.018 & 0.062 & -0.294 & 0.769 & \\
population:problem number & -0.003 & 0.062 & -0.052 & 0.959 & \\
source:information type:population & 0.052 & 0.092 & 0.570 & 0.569 & \\
source:population:problem number & 0.035 & 0.062 & 0.559 & 0.576 & \\
\hline
\end{tabular}

interactions between source, problem number, and population. Participant identity, age, and gender were included as random intercepts. Model outputs are shown in Table 515.

\subsubsection{Repeating behaviour for Stage A and Stage B}

Model fixed effects were source, information type, age, stage, population, and all interactions, with random intercepts for participant identity and problem number. Model outputs are in Table S16, 
Table S15: Experiment 1-2 combined data task success for Stage A Problems 1-5 for the older children.

\begin{tabular}{lrrrrr}
\hline & $b$ & $\mathrm{SE}(b)$ & \multicolumn{1}{c}{$\mathrm{z}$} & $\mathrm{p}$ & \\
\hline Intercept & 1.895 & 0.187 & 10.143 & $<0.001$ & $* * *$ \\
source & 0.090 & 0.156 & 0.580 & 0.562 & \\
information type & -1.650 & 0.159 & -10.405 & $<0.001$ & $* * *$ \\
population & 0.202 & 0.162 & 1.248 & 0.212 & \\
problem number & 0.430 & 0.079 & 5.450 & $<0.001$ & $* * *$ \\
source:information type & -0.006 & 0.136 & -0.042 & 0.967 & \\
source:population & -0.253 & 0.157 & -1.608 & 0.108 & \\
information type:population & -0.258 & 0.136 & -1.900 & 0.057 &. \\
source:problem number & -0.028 & 0.075 & -0.368 & 0.713 & \\
population:problem number & 0.195 & 0.076 & 2.569 & 0.010 & $*$ \\
source:information type:population & -0.015 & 0.136 & -0.109 & 0.913 & \\
source:population:problem number & -0.011 & 0.075 & -0.150 & 0.881 & \\
\hline
\end{tabular}

As there was a significant interaction between source, information type, age, and population, we split our dataset into younger and older children based on median age and repeated our analysis on these subsets. See Figure 56 for mean proportion of repeats by source and information type for each age group in each population.

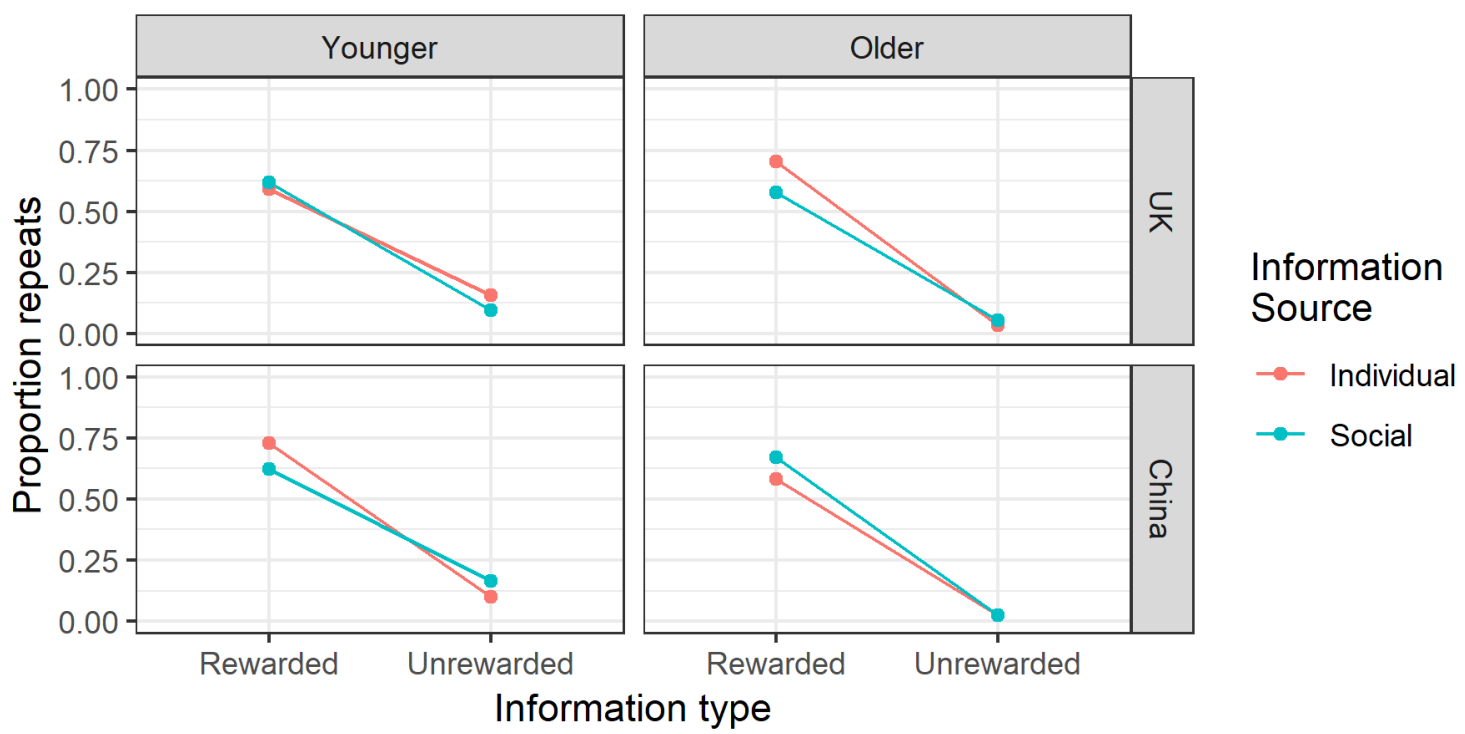

Figure S6: Experiments 1-2 mean repeating behaviour for Stage A and Stage B by age group, source, information type, and population.

For the younger children, model fixed effects were source, information type, stage, and population. Participant identity, problem number, and age were included as random intercepts. Model outputs are shown in Table S17. 
Table S16: Experiment 1-2 combined data repeating behaviour for Stage A and Stage B.

\begin{tabular}{|c|c|c|c|c|c|}
\hline & $b$ & $\operatorname{SE}(b)$ & z & $p$ & \\
\hline Intercept & -1.296 & 0.109 & -11.879 & $<0.001$ & $* * *$ \\
\hline source & 0.052 & 0.093 & 0.558 & 0.577 & \\
\hline information type & 1.995 & 0.061 & 32.642 & $<0.001$ & $* * *$ \\
\hline age & -1.219 & 0.235 & -5.182 & $<0.001$ & $* * *$ \\
\hline stage & -0.233 & 0.108 & -2.150 & 0.032 & $*$ \\
\hline population & -0.043 & 0.093 & -0.465 & 0.642 & \\
\hline source:information type & 0.047 & 0.058 & 0.802 & 0.423 & \\
\hline source:age & -0.047 & 0.235 & -0.200 & 0.841 & \\
\hline information type:age & 1.161 & 0.141 & 8.206 & $<0.001$ & $* * *$ \\
\hline source:stage & 0.056 & 0.107 & 0.518 & 0.605 & \\
\hline information type:stage & 0.757 & 0.107 & 7.058 & $<0.001$ & $* * *$ \\
\hline age:stage & 0.135 & 0.257 & 0.523 & 0.601 & \\
\hline source:population & -0.054 & 0.093 & -0.579 & 0.563 & \\
\hline information type:population & 0.156 & 0.058 & 2.669 & 0.008 & $* *$ \\
\hline age:population & -0.241 & 0.235 & -1.024 & 0.306 & \\
\hline stage:population & -0.126 & 0.107 & -1.172 & 0.241 & \\
\hline source:information type:age & -0.096 & 0.141 & -0.681 & 0.496 & \\
\hline source:information type:stage & -0.014 & 0.107 & -0.134 & 0.893 & \\
\hline source:age:stage & 0.016 & 0.257 & 0.061 & 0.951 & \\
\hline information type:age:stage & 0.600 & 0.256 & 2.342 & 0.019 & $*$ \\
\hline source:information type:population & -0.017 & 0.058 & -0.288 & 0.773 & \\
\hline source:age:population & -0.158 & 0.235 & -0.672 & 0.502 & \\
\hline information type:age:population & -0.019 & 0.142 & -0.134 & 0.894 & \\
\hline source:stage:population & -0.108 & 0.107 & -1.006 & 0.315 & \\
\hline information type:stage:population & 0.225 & 0.107 & 2.106 & 0.035 & $*$ \\
\hline age:stage:population & -0.074 & 0.257 & -0.286 & 0.775 & \\
\hline source:information type:age:stage & -0.355 & 0.256 & -1.387 & 0.166 & \\
\hline source:information type:age:population & -0.510 & 0.142 & -3.600 & $<0.001$ & $* * *$ \\
\hline source:information type:stage:population & -0.057 & 0.107 & -0.531 & 0.595 & \\
\hline source:age:stage:population & -0.013 & 0.257 & -0.049 & 0.961 & \\
\hline information type:age:stage:population & 0.231 & 0.256 & 0.903 & 0.366 & \\
\hline source:information type:age:stage:population & -0.017 & 0.256 & -0.065 & 0.948 & \\
\hline
\end{tabular}

For the older children, model fixed effects were source, information type, stage, and population. Participant identity and problem number were included as random intercepts. Model outputs are shown in Table 518 .

As there are significant interactions between source, information type, and population in the analyses of both the younger and the older age groups, we also repeated separate analyses on each of the younger UK, older UK, younger China, and older China subsets. For the UK data, this is equivalent to that reported in Supplemental Material Section 2.1.3; see Tables 56 and 57 .

For the younger China children, model fixed effects were source, information type, stage, 
Table S17: Experiments 1-2 combined repeating behaviour for Stage A and Stage B for the younger children.

\begin{tabular}{lrrrrr}
\hline & \multicolumn{1}{c}{$b$} & $\mathrm{SE}(b)$ & \multicolumn{1}{c}{$\mathrm{z}$} & $\mathrm{p}$ & \\
\hline Intercept & -0.823 & 0.127 & -6.452 & $<0.001$ & $* * *$ \\
source & 0.061 & 0.121 & 0.506 & 0.613 & \\
information type & 1.556 & 0.066 & 23.614 & $<0.001$ & $* * *$ \\
stage & -0.166 & 0.114 & -1.456 & 0.145 & \\
population & 0.092 & 0.123 & 0.750 & 0.453 & \\
source:information type & 0.053 & 0.062 & 0.859 & 0.390 & \\
source:stage & 0.058 & 0.114 & 0.512 & 0.608 & \\
information type:stage & 0.508 & 0.113 & 4.477 & $<0.001$ & $* * *$ \\
source:population & -0.037 & 0.122 & -0.307 & 0.759 & \\
information type:population & 0.161 & 0.063 & 2.573 & 0.010 & $*$ \\
stage:population & -0.081 & 0.114 & -0.711 & 0.477 & \\
source:information type:stage & 0.202 & 0.113 & 1.788 & 0.074 &. \\
source:information type:population & 0.203 & 0.062 & 3.272 & 0.001 & $* *$ \\
source:stage:population & -0.096 & 0.114 & -0.840 & 0.401 & \\
information type:stage:population & 0.160 & 0.113 & 1.422 & 0.155 & \\
source:information type:stage:population & -0.061 & 0.113 & -0.541 & 0.589 & \\
\hline
\end{tabular}

Table S18: Experiments 1-2 combined repeating behaviour for Stage A and Stage B for the older children.

\begin{tabular}{lrrrrr}
\hline & \multicolumn{1}{c}{$b$} & $\mathrm{SE}(b)$ & \multicolumn{1}{c}{$\mathrm{z}$} & \multicolumn{1}{c}{$\mathrm{p}$} & \\
\hline Intercept & -1.674 & 0.167 & -10.021 & $<0.001$ & $* * *$ \\
source & 0.004 & 0.139 & 0.026 & 0.979 & \\
information type & 2.347 & 0.100 & 23.435 & $<0.001$ & $* * *$ \\
stage & -0.333 & 0.176 & -1.894 & 0.058 &. \\
population & -0.204 & 0.139 & -1.470 & 0.142 & \\
source:information type & 0.057 & 0.095 & 0.594 & 0.552 & \\
source:stage & 0.077 & 0.174 & 0.445 & 0.657 & \\
information type:stage & 1.005 & 0.174 & 5.769 & $<0.001$ & $* * *$ \\
source:population & -0.100 & 0.139 & -0.722 & 0.470 & \\
information type:population & 0.181 & 0.095 & 1.905 & 0.057 &. \\
stage:population & -0.158 & 0.174 & -0.908 & 0.364 & \\
source:information type:stage & -0.215 & 0.174 & -1.238 & 0.216 & \\
source:information type:population & -0.201 & 0.095 & -2.111 & 0.035 & $*$ \\
source:stage:population & -0.063 & 0.174 & -0.362 & 0.717 & \\
information type:stage:population & 0.278 & 0.174 & 1.600 & 0.110 & \\
source:information type:stage:population & -0.076 & 0.174 & -0.438 & 0.661 & \\
\hline
\end{tabular}

and all interactions. Participant identity, gender, and age were included as random intercepts. Model outputs are shown in Table S19.

For the older China children, model fixed effects were source, information type, stage, 
Table S19: Experiments 1-2 combined repeating behaviour for Stage A and Stage B for the younger China children.

\begin{tabular}{lrrrrr}
\hline & $b$ & $\mathrm{SE}(b)$ & \multicolumn{1}{c}{$\mathrm{z}$} & \multicolumn{1}{c}{$\mathrm{p}$} & \\
\hline Intercept & -0.767 & 0.492 & -1.559 & 0.119 & \\
source & 0.004 & 0.202 & 0.019 & 0.985 & \\
information type & 1.832 & 0.109 & 16.793 & $<0.001$ & $* * *$ \\
stage & -0.271 & 0.175 & -1.544 & 0.123 & \\
source:information type & 0.254 & 0.102 & 2.496 & 0.013 & $*$ \\
source:stage & -0.040 & 0.175 & -0.226 & 0.821 & \\
information type:stage & 0.692 & 0.175 & 3.951 & $<0.001$ & $* * *$ \\
source:information type:stage & 0.140 & 0.175 & 0.804 & 0.422 & \\
\hline
\end{tabular}

and all interactions. Participant identity, problem number, and age were included as random intercepts. Model outputs are shown in Table $\$ 20$.

Table S20: Experiments 1-2 combined repeating behaviour for Stage A and Stage B for the older China children.

\begin{tabular}{lrrrrr}
\hline & $b$ & $\mathrm{SE}(b)$ & $\mathrm{z}$ & \multicolumn{1}{c}{$\mathrm{p}$} & \\
\hline Intercept & -1.907 & 0.259 & -7.367 & $<0.001$ & $* * *$ \\
source & -0.101 & 0.223 & -0.451 & 0.652 & \\
information type & 2.578 & 0.164 & 15.681 & $<0.001$ & $* * *$ \\
stage & -0.492 & 0.288 & -1.708 & 0.088 &. \\
source:information type & -0.144 & 0.157 & -0.923 & 0.356 & \\
source:stage & 0.011 & 0.285 & 0.038 & 0.969 & \\
information type:stage & 1.296 & 0.286 & 4.533 & $<0.001$ & $* * *$ \\
source:information type:stage & -0.285 & 0.285 & -0.997 & 0.319 & \\
\hline
\end{tabular}

\subsection{Experiment 3}

\subsubsection{Task success for Stage A (all problems)}

In contrast to Experiments $1-2$, most of the 184 children completed all 10 Stage A problems without achieving five consecutive successful TTs (154 children: 84\%). Only 30 (16\%; 15 in each condition) reached the criterion. Seven [4\%] reached criterion in 5 problems, 2 [1\%] in 6 problems, 2 [1\%] in 7 problems, 5 [3\%] in 8 problems, 2 [1\%] in 9 problems, and 12 [7\%] in 10 problems. Therefore in contrast to our approach for Experiments 1-2 (analysing success by using only the first five problems of Stage A), here we use all of the Stage A data.

Note that we preregistered the wrong fixed effects for our analysis here (we stated that our fixed effects would be problem number, stage, age, source, information type, and the interactions of age and source, and age and information type; see https://osf .io/qtpnm/). Our 
approach here is instead one consistent with that in Experiments 1-2. Repeating our analysis with the preregistered fixed effects does not alter the pattern of results or the conclusions we draw from the data.

Model fixed effects were source, information type, age, and problem number, all interactions between source, information type, and age, and the interaction between source and problem number. Participant identity was included as a random intercept. Model outputs are in Table $\mathbf{S 2 1}$.

Table S21: Experiment 3 task success for Stage A.

\begin{tabular}{lrrrrr}
\hline & $b$ & $\mathrm{SE}(b)$ & \multicolumn{1}{c}{$\mathrm{z}$} & \multicolumn{1}{c}{$\mathrm{p}$} & \\
\hline Intercept & -0.244 & 0.078 & -3.122 & 0.002 & $* * *$ \\
source & 0.085 & 0.078 & 1.084 & 0.279 & \\
information type & 0.886 & 0.060 & 14.721 & $<0.001$ & $* * *$ \\
age & 0.426 & 0.196 & 2.173 & 0.030 & $*$ \\
problem number & 0.035 & 0.020 & 1.783 & 0.075 &. \\
source:information type & -0.005 & 0.057 & -0.086 & 0.931 & \\
source:age & -0.187 & 0.196 & -0.954 & 0.340 & \\
information type:age & 1.361 & 0.146 & 9.317 & $<0.001$ & $* * *$ \\
source:problem number & -0.025 & 0.020 & -1.292 & 0.196 & \\
source:information type:age & 0.229 & 0.142 & 1.611 & 0.107 & \\
\hline
\end{tabular}

\subsubsection{Repeating behaviour for Stage A (all problems)}

Our fixed effects model structure was the same for Experiment 1 (Supplemental Material Section 2.1.3, except that, as we only consider the Stage A data here, there are no fixed effects involving stage. That is, our fixed effects were source, information type, age, and all interactions. Participant identity, experimenter identity, participant gender, and problem number were included as random intercepts. Model outputs are in Table $\$ 22$.

Table S22: Experiment 3 repeating behaviour for Stage A.

\begin{tabular}{lrrrrr}
\hline & $b$ & $\mathrm{SE}(b)$ & \multicolumn{1}{c}{$\mathrm{z}$} & \multicolumn{1}{c}{$\mathrm{p}$} & \\
\hline Intercept & -1.037 & 0.195 & -5.328 & $<0.001$ & $* * *$ \\
source & -0.014 & 0.123 & -0.117 & 0.907 & \\
information type & 0.339 & 0.061 & 5.564 & $<0.001$ & $* * *$ \\
age & -1.529 & 0.312 & -4.905 & $<0.001$ & $* * *$ \\
source:information type & -0.082 & 0.061 & -1.352 & 0.176 & \\
source:age & -0.278 & 0.306 & -0.911 & 0.362 & \\
information type:age & -0.518 & 0.153 & -3.379 & $<0.001$ & $* * *$ \\
source:information type:age & 0.181 & 0.153 & 1.181 & 0.238 & \\
\hline
\end{tabular}




\subsection{Experiments $1-3$ combined data}

To investigate any differences in task success and proportion of repeats between the reward structure of Experiments 1-2 and that of Experiment 3, we combined the Stage A problems 1-5 data for all Experiments 1-3 and repeated the analyses with reward structure as an additional variable. Note that for meaningful comparison between the Experiments 1-2 and Experiment 3 data for the repeats measure, we excluded the Stage A Problems 6-10 data from the Experiment 3 data here.

\subsubsection{Task success for Stage A Problems 1-5}

Our fixed effects were source, information type, age, problem number, reward structure, all interactions between source, information type, age, and reward structure, and all interactions between source, problem number, and reward structure. We included participant identity as a random intercept. Model outputs are in Table $\$ 23$.

Table S23: Experiment 1-3 combined data task success for Stage A Problems 1-5.

\begin{tabular}{lrrrrr}
\hline & $b$ & $\mathrm{SE}(b)$ & $\mathrm{z}$ & $\mathrm{p}$ & \\
\hline Intercept & 0.584 & 0.071 & 8.185 & $<0.001$ & $* * *$ \\
source & 0.099 & 0.070 & 1.401 & 0.161 & \\
information type & -0.135 & 0.060 & -2.249 & 0.025 & $*$ \\
age & 0.722 & 0.177 & 4.074 & $<0.001$ & $* * *$ \\
reward structure & 0.951 & 0.073 & 12.979 & $<0.001$ & $* * *$ \\
problem number & 0.179 & 0.037 & 4.791 & $<0.001$ & $* * *$ \\
source:information type & -0.016 & 0.060 & -0.259 & 0.795 & \\
source:age & 0.180 & 0.177 & 1.017 & 0.309 & \\
information type:age & 0.030 & 0.150 & 0.201 & 0.840 & \\
source:reward structure & -0.048 & 0.070 & -0.682 & 0.495 & \\
information type:reward structure & -1.093 & 0.065 & -16.873 & $<0.001$ & $* * *$ \\
age:reward structure & 0.321 & 0.177 & 1.817 & 0.069 &. \\
source:problem number & -0.057 & 0.037 & -1.523 & 0.128 & \\
reward structure:problem number & 0.106 & 0.037 & 2.859 & 0.004 & $* *$ \\
source:information type:age & 0.080 & 0.150 & 0.536 & 0.592 & \\
source:information type:reward structure & -0.071 & 0.060 & -1.189 & 0.234 & \\
source:age:reward structure & 0.128 & 0.177 & 0.725 & 0.469 & \\
information type:age:reward structure & -1.512 & 0.153 & -9.877 & $<0.001$ & $* * *$ \\
source:reward structure:problem number & 0.045 & 0.037 & 1.200 & 0.230 & \\
source:information type:age:reward structure & 0.022 & 0.150 & 0.143 & 0.886 & \\
\hline
\end{tabular}

There was a main effect of reward structure, such that children were more successful with the congruent reward structure (Experiments 1-2), compared with the reversed reward structure (Experiment 3$)(p<.001)$. There was also an interaction between reward structure and 
information type $(p<.001)$, consistent with the higher lose-shift versus win-stay success in Experiments 1-2, and the higher win-shift versus lose-stay success in Experiment 3. This interaction was more pronounced in older children (information type $\mathrm{x}$ age $\mathrm{x}$ reward structure: $p<.001)$. The effects of age $(p<.001)$ and problem number $(p<.001)$ in the analyses for Experiments 1-2 were replicated here, with the effect of problem number more pronounced in the congruent reward structure of Experiments 1-2 (reward structure x problem number: $p=$ $.004)$. There was no effect of source $(p=.161)$, nor any significant interactions involving source $(p \geq .128)$.

\subsubsection{Repeating behaviour for Stage A Problems 1-5}

Our fixed effects were source, information type, age, reward structure, and all interactions. We included random intercepts for participant identity, experimenter identity, and problem number. Model outputs are in Table 524

Table S24: Experiment 1-3 combined data repeating behaviour for Stage A.

\begin{tabular}{lrrrrr}
\hline & $b$ & $\mathrm{SE}(b)$ & $\mathrm{z}$ & $\mathrm{p}$ & \\
\hline Intercept & -1.128 & 0.170 & -6.615 & $<0.001$ & $* * *$ \\
source & -0.074 & 0.081 & -0.914 & 0.361 & \\
information type & 1.000 & 0.066 & 15.149 & $<0.001$ & $* * *$ \\
age & -1.628 & 0.207 & -7.854 & $<0.001$ & $* * *$ \\
reward structure & -0.095 & 0.084 & -1.129 & 0.259 & \\
source:information type & -0.061 & 0.062 & -0.986 & 0.324 & \\
source:age & 0.048 & 0.204 & 0.234 & 0.815 & \\
information type:age & 0.319 & 0.158 & 2.025 & 0.043 & $*$ \\
source:reward structure & 0.004 & 0.081 & 0.051 & 0.960 & \\
information type:reward structure & 0.587 & 0.063 & 9.322 & $<0.001$ & $* * *$ \\
age:reward structure & 0.101 & 0.204 & 0.493 & 0.622 & \\
source:information type:age & 0.140 & 0.158 & 0.891 & 0.373 & \\
source:information type:reward structure & 0.082 & 0.062 & 1.326 & 0.185 & \\
source:age:reward structure & 0.157 & 0.203 & 0.774 & 0.439 & \\
information type:age:reward structure & 0.716 & 0.158 & 4.536 & $<0.001$ & $* * *$ \\
source:information type:age:reward structure & 0.141 & 0.158 & 0.896 & 0.370 & \\
\hline
\end{tabular}

Again, there was no evidence of an effect of source $(p=.361)$. There was no effect of reward structure on overall proportions of repeats $(p=.259)$, although there was an interaction between reward structure and information type, such that the effect of information type was stronger with the congruent reward structure, compared to when it was reversed $(p<.001)$. Older children overall repeated less than younger $(p<.001)$, and this was more pronounced following unrewarded ITs (information type $x$ age: $p=.043$ ), particularly when the reward structure was congruent (information type $x$ age $x$ reward structure: $p<.001$ ). 


\section{Modelling Participant Biases in Experiments 1 and 2}

We analysed the results of Experiments 1-2 using a simple mathematical model of domaingeneral learning by statical inference. This provided a quantitative benchmark against which participants' selections could be evaluated, and helped to illuminate potential biases underpinning behaviour in the task.

\subsection{Method: Model details}

We computed the probability of repeating an IT selection under a Bayesian model of ideal statistical learning and decision making in this context. Each individual TT selection can be understood as a binomial trial in which repeating the IT selection results in task success (with probability $p$ ) or failure (with probability $1-p$ ). After observing $i$ rewarded ITs, an ideal statistical learner's expectations of the reward structure can be modelled using a Beta distribution with parameters $\alpha+i$ and $\beta$; after observing $j$ unrewarded ITs, an ideal statistical learner's expectations can be modelled using a Beta distribution with parameters $\alpha$ and $\beta+j$.

We analysed two versions of this model. In both cases we analysed rewarded and unrewarded trials independently. We focused on analysing the decisions of older children (4yo or older) because these data represent a large but clean data subset in which signatures of withintask learning effects are clearest. Including younger children's decisions in our analysis does not alter our conclusions. Decision theoretic considerations suggest that there is an optimal way to respond to the explore-exploit trade-off inherent to this task: take a random sample $(\hat{p})$ from the posterior distribution for $p$, and repeat the IT selection if the sampled value is larger than $1 / 2$. This is a simple example of a strategy known as Thompson sampling. An alternative to this strategy, known as Probability Matching, is to repeat the IT selection with probability $\hat{p}$. Probability matching is more exploratory than Thompson sampling.

These two strategies provide quantitative benchmarks against which we can compare children's performance in the task. Both models include bias parameters $\alpha$ and $\beta$. The ratio of these parameters determines whether the model is inherently biased towards repeating $(\beta / \alpha<1)$ or deviating $(\beta / \alpha>1)$ from IT selections. In this context, a bias in the model can be understood to characterise the rate at which disconfirming trials overturn a favoured reward structure. Figure 57 shows the level of task success predicted by both models, assuming unbiased parametrisation ( $\beta / \alpha=1$, top row), and after inferring model-specific maximumlikelihood estimates for $\beta / \alpha$ (bottom row). In unbiased form, both models (a) fail to predict the initial asymmetry in task success between rewarded and unrewarded ITs, (b) over-predict task success following rewarded IT selections, and (c) under-predict task success following unrewarded IT selections. 

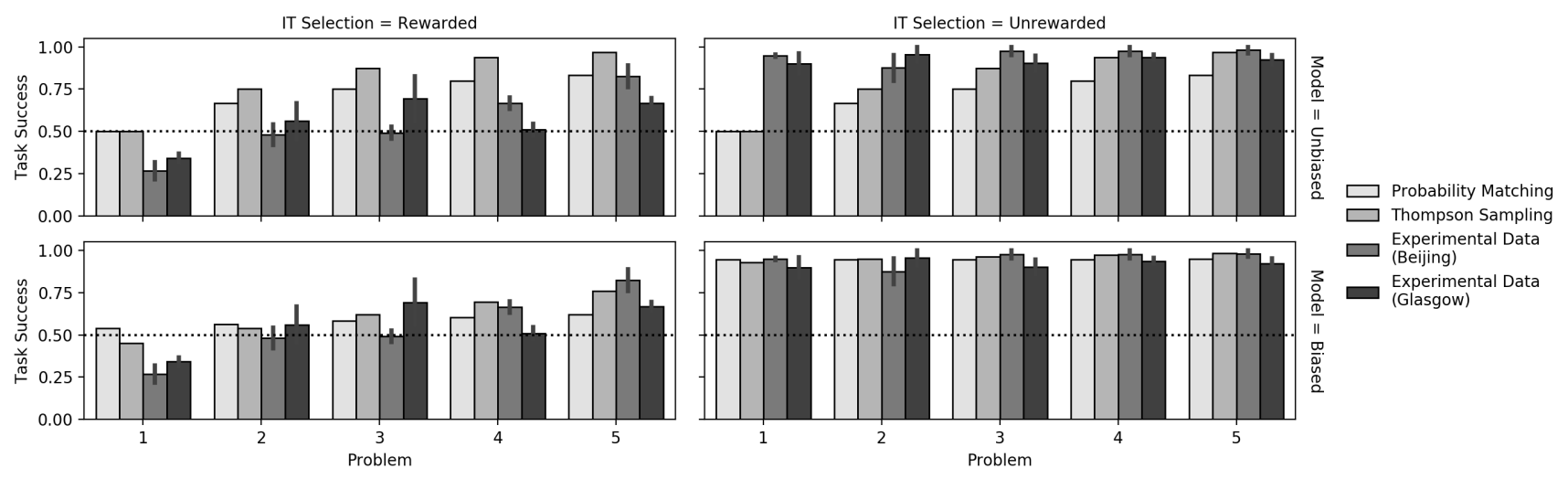

Figure S7: Task success rates under two models of domain-general learning (Thompson sampling and Probability Matching). Neutral bias parameters (top) and after fitting bias parameters to the experimental data (bottom), alongside task success among older participants (children aged 4 and above) in Experiments 1-2.

\subsection{Results: Inferred biases}

Both models capture task performance relatively well after fitting the bias parameter $\beta / \alpha$. We found the Thompson Sampling model to be a marginally better fit to the data. To investigate the biases of the model further, we characterised the log-likelihood surface for the experimental data under the Thompson Sampling model. Figure $\$ 8$ shows the log-likelihood surface over the logarithm of the ratio of the bias parameters: $\ln (\beta / \alpha)$. We calculated this surface looking at decisions in the Social information condition (blue), the Individual information condition (orange), or both (green), accounting for just the first (left), the first and the second (middle), or the first, second, and third (right) trials following rewarded (top) or unrewarded (bottom) IT selections. Here, above, and in the analyses presented in the main text, the log-likelihood of the experimental data is calculated by setting $\alpha=10$ and varying the ratio $\beta / \alpha$, looking at the decisions of older (aged 4 and above) participants in Stage A of Experiments 1-2.

In all cases, the maximum likelihood estimate for $\ln (\beta / \alpha)$ is larger than zero, consistent with a robust bias against repeating the IT selection, accounting for participants' observation history. This bias is stronger following unrewarded compared to rewarded IT selections. In the context of rewarded ITs, our estimate of the bias gets weaker the more decisions we account for. Most strikingly, across all contexts, the MLE estimate of this bias is almost identical in the Social and Individual conditions.

In summary, we observed a correspondence between participants' decisions and a widely studied domain-general model of learning and decision making, but only when this model is initially biased against repeating prior behaviour. Our estimates of this bias are consistent with a win-stay lose-shift pattern of decision making, which is consistent across both information conditions. 

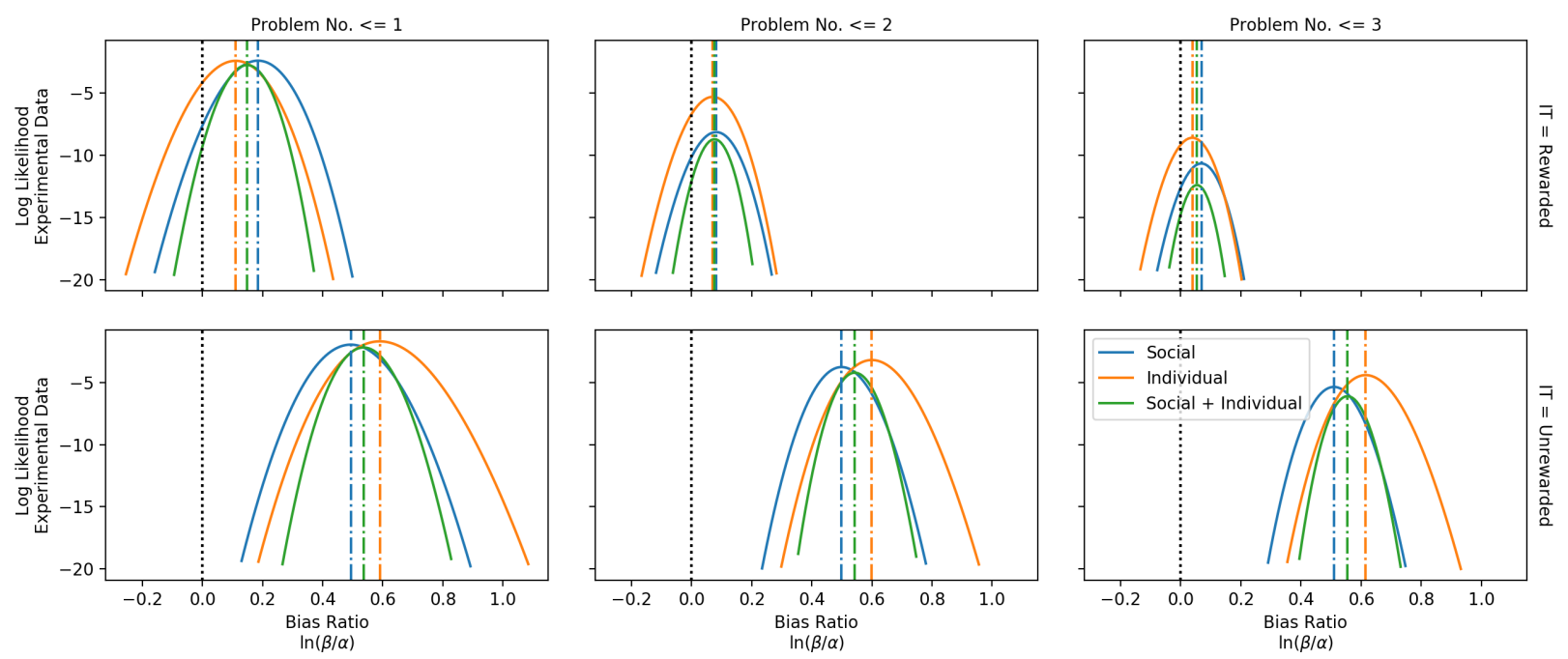

Figure S8: Log likelihood of the experimental data (participants over 3yo in Experiments 1-2) under the Thompson Sampling model of learning in this task. $\ln (\beta / \alpha)=0$ is unbiased. $\ln (\beta / \alpha)>0$ represents a bias against repeating IT selections.

\section{Additional methodological details for Experiments 1-3}

\subsection{Data collection plan}

In Experiments 1 and 2, we aimed to collect Stage A and Stage B data from a minimum of 320 children: 20 children for each of the age groups of 2 and under, 3, 4, and 5 (whole years), for each condition (Individual and Social), and for each population (UK and China). Our sample size was determined by estimating the maximum number of children we would be able to test given constraints on personnel and the availability of suitable testing time at the science centre in Glasgow and the kindergarten in Beijing, while ensuring that we would have at least comparable numbers for each age group, in each condition and population, as those of related studies involving young children (e.g. Lyons et al., 2007; Dean et al., 2012; Flynn et al., 2016). We anticipated that obtaining data for the 2 and under age groups would be more difficult (due to factors such as lower attention spans, wariness of the experimenter, and parental interference), and this is reflected in our slightly lower sample sizes for these groups (see below). Where a child completed Stage A but not B, the child was not counted toward our target sample size, but their Stage $A$ data was retained and included in our analysis (this was the case for the data of 12 children in our total sample of 331).

In Experiment 3, we aimed to collect Stage A and Stage B data from 160 children: a minimum of 20 children for each of the ages 3, 4, and 5, for each condition (Individual and Social). We aimed to collect data from as many 2-year-olds as possible while collecting the rest of the data, though as in Experiments 1-2, anticipated a slightly smaller sample size in our youngest age group. Again, where a child completed Stage $A$ but not $B$, the child was not counted toward our target sample size, but their Stage A data was retained and included in our analysis (this 
was the case for the data of 15 children in our sample of 184).

\subsection{Data exclusion criteria}

Prior to data collection, we determined the possible cases where it would be necessary for a child's data to be entirely excluded from our analyses: (1) if the child, parent, or guardian wanted to stop their participation before they had completed Stage A of the experiment; (2) there was a technological problem, either when running the experiment (e.g. the tablet ran out of power) or in saving the data; (3) there was an experimenter error (e.g. deviating from the prepared oral instructions; see below); (4) the child received task assistance (e.g. by receiving advice from a parent, guardian, or sibling; this was only relevant for Experiments 1 and 3). We made the decision to exclude all of the child's data in these cases in the interests of controlling for unwanted influences as much as possible. We could, for example, have only removed the data for the specific problem where a child received parental assistance, but excluded all of their data in case that assistance influenced the child's behaviour on subsequent problems. Where a child's data was excluded, we did not count that child toward our target sample size (i.e. another child was recruited in their place).

\subsection{Data collected}

\subsubsection{Experiments 1-2}

\subsubsection{UK}

172 children were recruited at Glasgow Science Centre (http: //www . glasgows ciencecentre. org/). Of these, 2 ( 1 female; 1 male) were 1 year old, 33 (19 female; 14 male) were 2 years old, 48 ( 22 female; 26 male) were 3 years old, 47 ( 31 female; 16 male) were 4 years old, and 42 ( 26 female; 16 male) were 5 years old. An additional 18 children ( 1 x 1-year-old, 8 × 2 -year-old, $6 \times 3$-year-old, $2 \times 4$-year-old, and $1 \times 5$-year-old) were recruited, but were excluded due to their fulfilling at least one of the criteria described above. Testing took place at a table, with the child sat next to the experimenter. The area was in a public space, but separated from other visitors to the Centre by a desk. Younger or less confident children were accompanied in the testing area by a parent or guardian, who was instructed not to provide the child with any assistance relevant to the task. Written informed consent was obtained from the child's parent or guardian prior to their taking part in the study. The child was given a sticker at the end, regardless of performance and task completion.

\subsubsection{China}

159 children were recruited at a kindergarten in Beijing. Of these, 31 (17 female; 14 male) were 2 years old, 42 ( 21 female; 21 male) were 3 years old, 44 ( 24 female; 20 male) were 4 years old, and 42 ( 21 female; 21 male) were 5 years old. An additional 14 children ( 7 x 2-year old, 4 x 
3-year old, $1 \times 4$-year old, and $2 \times 5$-year old) were recruited, but were excluded as above. Testing took place in a quiet area, with the child sat next to the experimenter. Written informed consent was obtained from the child's parent or guardian prior to their taking part in the study.

\subsubsection{Experiment 3}

184 children were recruited at Glasgow Science Centre. Of these, 1 (male) was 1 year old, 33 (19 female; 14 male) were 2 years old, 49 ( 29 female; 20 male) were 3 years old, 49 (26 female; 23 male) were 4 years old, and 52 ( 29 female; 23 male) were 5 years old. An additional 32 children (10 $\times 2$-year old, $9 \times 3$-year old, $4 \times 4$-year old, and $9 \times 5$-year old) were recruited, but were excluded as above.

\subsubsection{Children aged 6 and above}

While collecting the data for Experiments 1 and 3 at Glasgow Science Centre, we often had children aged 6 and above interested in our research, and occasionally we allowed particularly keen ones (usually older siblings of our participants) to try the task themselves. Their responses were automatically recorded, but as these children were not part of our original target sample we excluded their data from all of our analyses. There is also too little additional data from these older children to consider including them in any exploratory analyses (there was only completely data from an additional 12 children in Experiment 1 and 9 children in Experiment 2, with unequal numbers of each age and in each condition).

\subsection{Additional design details}

The experiment was written in PsychoPy 1.83 (Peirce et al. 2019) and run on a Microsoft Surface 3.

At the start of the experiment (in both conditions), the child was told: "We're going to play a game. We're looking for a monkey, but the monkey's hiding. Let's look for him." In the IT in the Social condition, the experimenter said "My turn", before making the selection; in the IT in the Individual condition, the experimenter encouraged the child to make a selection with "Look for the monkey". In the TT (in both conditions), the experimenter encouraged the child to make a selection with "Where's the monkey?" In all ITs and TTs, the experimenter responded to all rewarded selections with "There he is!", and all unrewarded selections with "Oh no, no monkey".

In Stage A problems, one stimulus was on the left of the screen and one on the right. In Stage B problems, one stimulus was on the left of the screen, one in the centre, and one on the right. Each stimulus was randomly generated to have 3, 4, 5, or 6 sides, fill-coloured using a randomly-generated RGB colour code, and white bordered. These stimuli were randomly 
generated for each problem, to reduce the effect of any attentional biases due to reoccurring stimuli being previously associated with a reward (as discussed in Anderson et al., 2013).

To make the pairing of the information and test trials salient, each problem was preceded by a 1-second blank-screen time out, and the stimuli scrolled in from the top of the screen over 2 seconds before the information trial, and scrolled out to the bottom of the screen over 2 seconds after the test trial. At the start of each problem from the second problem onwards, the experimenter said "Now let's try another one".

In each of Stage A and Stage B, five of the ITs were rewarded and five unrewarded, in a randomised order for each child. Whether an IT was to be rewarded or unrewarded was therefore predetermined for each problem, rather than dependent on which stimulus was selected in the IT. If the IT was rewarded and the child selected the left stimulus, for example, then the monkey would be located on the left side; if they selected the right stimulus, the monkey would be located on the right side.

Stage A ended following five consecutively rewarded TTs (to maintain engagement in children who demonstrated high proficiency), or at the maximum of 10 problems. A correct selection in a TT would reveal the monkey with the accompanying sound cue, after which an image of a cartoon banana was added at the top of the screen. In Stage A, if the child made the incorrect choice in the TT, any bananas already displayed were removed; as such the bananas indicated the number of consecutively rewarded test trials. Bananas were not removed following an unrewarded test trial in Stage $B$, however.

At the end of Stage $A$, the researcher checked that the child was happy to continue to Stage $B$ by asking "Would you like to play some more?"

\subsection{Participant breakdown by age and condition}

Participants by age (whole years) and condition for Experiments 1-3 is in Table S25.

\section{Experiment 4: effect of non-differential feedback}

This study was an adaptation of Experiments 1-3 aiming to establish children's biases relevant to source and type in the absence of feedback, i.e. unsuccessful selections made in the test trials of earlier problems influencing later behaviour. The children therefore received indiscriminate feedback; their test trial selections revealed the reward regardless of whether they provided a repeat or non-repeat response, and regardless of whether the information trial was rewarded or unrewarded. 
Table S25: Participants by age (whole years) and condition for Experiments 1-3. Number before brackets indicates number of participants who at least completed Stage $A$; in brackets is the number who completed both Stage A and Stage B.

\begin{tabular}{|c|c|c|c|c|}
\hline Experiment 1 & $\leq 2$ уо & 3уо & 4 уо & 5 yо \\
\hline Individual & $17(15)$ & $24(20)$ & $23(23)$ & $22(21)$ \\
\hline Social & $18(17)$ & $24(22)$ & $24(24)$ & $20(20)$ \\
\hline Total & $35(32)$ & $48(42)$ & $47(47)$ & $42(41)$ \\
\hline Experiment 2 & 2 yo & 3уо & 4 yo & 5 yo \\
\hline Individual & $14(14)$ & $22(22)$ & $20(20)$ & $21(20)$ \\
\hline Social & 17 (17) & $20(20)$ & $24(24)$ & $21(20)$ \\
\hline Total & $31(31)$ & $42(42)$ & $44(44)$ & $42(40)$ \\
\hline Experiment 3 & $\leq 2$ yо & 3уо & 4 yo & 5 yo \\
\hline Individual & $16(13)$ & $24(23)$ & $25(24)$ & $26(24)$ \\
\hline Social & $18(14)$ & $25(22)$ & $24(23)$ & $26(26)$ \\
\hline Total & $34(27)$ & $49(45)$ & $49(47)$ & $52(50)$ \\
\hline
\end{tabular}

\subsection{Results}

Repeating behaviour by information type (rewarded or unrewarded) and age, by source, is illustrated in Figure $\$ 9$.

Repetition rate was below chance $(M=0.24, S D=0.22, p<.001)$. In the Individual condition, average repeating behaviour was $0.41(S D=0.38)$ following rewarded ITs and $0.07(S D=0.16)$ following unrewarded ITs. In the Social condition, average repeating behaviour was 0.42 (SD = 0.41 ) following rewarded ITs and 0.09 (SD = 0.25) following unrewarded ITs.

We constructed a linear mixed model with logit link and repeating behaviour as dependent variable. Our fixed effects were source, information type, age, problem number, and all interactions. We included participant identity as a random intercept. Model outputs are in Table S26.

Repeats were less common following unrewarded information trials $(p<.001)$. There was no evidence of an effect of age $(p=.124)$, problem number $(p=.662)$, or source $(p=.890)$.

\subsection{Discussion}

Non-repeating behaviour was more common than repeating behaviour overall, though repeating behaviour was more likely following rewarded information trials compared to unrewarded information trials. There was no evidence that older children made different selections to younger, nor that there was any effect of source or of task experience (problem number). 


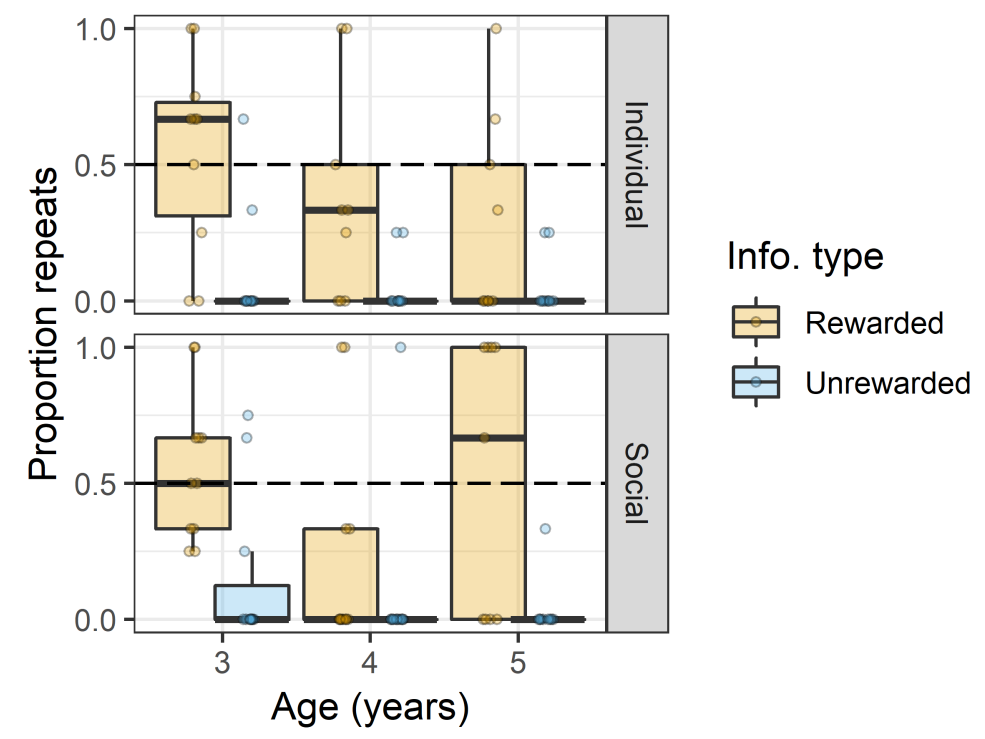

Figure S9: Proportion of repeats for each child by source (Individual or Social), and information type (rewarded or unrewarded). Each participant's performance is indicated by two points, as information type is a within-participant manipulation: one point is the proportion of repeats for rewarded problems, and one for unrewarded problems. Dashed lines indicate chance performance.

Table S26: Experiment 4 repeating behaviour.

\begin{tabular}{lrrrrr}
\hline & \multicolumn{1}{c}{$b$} & $\mathrm{SE}(b)$ & \multicolumn{1}{c}{$\mathrm{z}$} & \multicolumn{1}{c}{$\mathrm{p}$} & \\
\hline Intercept & -1.851 & 0.277 & -6.674 & $<0.001$ & $* * *$ \\
age & -1.212 & 0.787 & -1.539 & 0.124 & \\
information type & 1.332 & 0.186 & 7.163 & $<0.001$ & $* * *$ \\
source & -0.034 & 0.249 & -0.138 & 0.890 & \\
problem number & -0.036 & 0.081 & -0.437 & 0.662 & \\
age:information type & -0.109 & 0.536 & -0.204 & 0.838 & \\
age:source & -0.243 & 0.777 & -0.313 & 0.754 & \\
information type:source & 0.053 & 0.172 & 0.310 & 0.757 & \\
age:problem number & 0.220 & 0.252 & 0.871 & 0.383 & \\
information type:problem number & 0.110 & 0.085 & 1.305 & 0.192 & \\
source:problem number & 0.029 & 0.081 & 0.353 & 0.724 & \\
age:information type:source & -0.347 & 0.537 & -0.646 & 0.518 & \\
age:information type:problem number & 0.107 & 0.258 & 0.414 & 0.679 & \\
age:source:problem number & -0.093 & 0.252 & -0.368 & 0.713 & \\
information type:source:problem number & 0.056 & 0.084 & 0.662 & 0.508 & \\
age:information type:source:problem number & 0.236 & 0.259 & 0.914 & 0.361 & \\
\hline
\end{tabular}

This pattern of results mirrors that of Experiments 1-3, except that in the absence of feedback about a particular reward structure (e.g. the congruent reward structure of Experiments 1-2 or the reversed one of Experiment 3), we see no effect of age. Though this might be an effect of this study's smaller sample size ( $N=60$, cf. $N$ ranging from 159 to 189 in Experiments 
1-3) and having fewer problems per participant (7 cf. 10-20), there is no evidence here that differently aged children initially approach the task with different biases.

\subsection{Methods}

The design of this study followed that of Experiments 1-3, with each child being placed in either an Individual or Social condition and being presented with a series of problems, each consisting of an IT and a TT as before. This study differed from the earlier experiments in that there was only one stage, equivalent to Stage A in Experiments 1-3, with two stimuli for each problem, and a total of 7 problems. To control for any lasting influence of the first problem encountered by each child, the information trial for the initial problem was rewarded for half the children of each age, and unrewarded for the others. The remaining 6 problems were equally split between having rewarded and unrewarded information trials, presented in a random order.

In each test trial, the child's selection would always reveal the reward, regardless of the type of information trial and whether the child made a repeating or non-repeating selection.

\subsubsection{Participants}

We aimed to collect data from 60 children, or as close to this as possible in one week of data collection: a minimum of 10 children for each of the ages 3,4 , and 5, for each condition (Individual and Social). We aimed to collect data from as many 2-year-olds as possible while collecting the rest of the data, though as in Experiments 1 and 2, anticipated smaller sample sizes.

60 children were recruited at Glasgow Science Centre as in Experiments 1 and 3. Of these, 1 (female) was 2 years old, 20 (12 female; 8 male) were 3 years old, 21 (10 female; 11 male) were 4 years old, and 18 ( 6 female; 12 male) were 5 years old. An additional 7 children ( $1 \mathrm{x}$ 2-year old, $2 \times 3$-year old, $2 \times 4$-year old, and $2 \times 5$-year old) were recruited, but their data is excluded from the analysis as the child did not want to complete the experiment, or before they could do so, there was an experimenter error, or the child received task assistance from a parent.

Participants by age (whole years) and condition is in Table $\mathbf{S 2 7 .}$

Table S27: Participants by age (whole years) and condition for Experiment 4.

\begin{tabular}{cccc} 
& $\leq 3$ yo & 4 yo & 5 yo \\
\hline $\begin{array}{c}\text { Individual } \\
\text { Social }\end{array}$ & 10 & 9 & 9 \\
\hline Total & 21 & 12 & 9 \\
\hline
\end{tabular}




\section{References}

Anderson, B. A., Laurent, P. A., and Yantis, S. 2013. Reward predictions bias attentional selection. Front Hum Neurosci, 7(June):262.

Barr, D. J., Levy, R., Scheepers, C., and Tily, H. J. 2013. Random effects structure for confirmatory hypothesis testing: Keep it maximal. Journal of Memory and Language, 68(3):255-278.

Bates, D., Maechler, M., and Bolker, B. 2013. Ime4: Linear mixed-effects models using S4 classes.

Dean, L. G., Kendal, R. L., Schapiro, S. J., Thierry, B., and Laland, K. N. 2012. Identification of the Social and Cognitive Processes Underlying Human Cumulative Culture. Science, 335:1114-1118.

Flynn, E., Turner, C., and Giraldeau, L.-A. 2016. Selectivity in social and asocial learning: investigating the prevalence, effect and development of young children's learning preferences. Philosophical Transactions of the Royal Society B, 271:20150189.

Lyons, D. E., Young, A. G., and Keil, F. C. 2007. The hidden structure of overimitation. Proceedings of the National Academy of Sciences of the United States of America, 104(50):19751-19756.

Peirce, J., Gray, J. R., Simpson, S., MacAskill, M., Höchenberger, R., Sogo, H., Kastman, E., Lindel $\varnothing$ v, J. K., and Peirce, J. 2019. PsychoPy2: Experiments in behavior made easy. Behavior Research Methods, 51:195-203.

R Core Team 2013. R: A language and environment for statistical computing. Available at http://www.r-project.org/. 